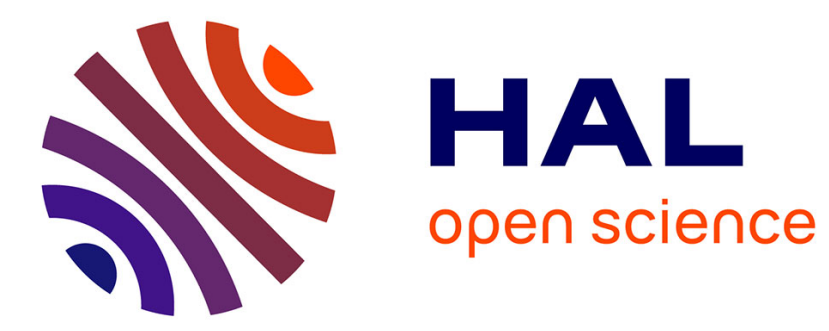

\title{
Multilayer four-flux matrix model accounting for directional-diffuse light transfers
}

Lionel Simonot, Roger D. Hersch, Mathieu Hébert, Serge Mazauric

\section{To cite this version:}

Lionel Simonot, Roger D. Hersch, Mathieu Hébert, Serge Mazauric. Multilayer four-flux matrix model accounting for directional-diffuse light transfers. Applied optics, 2016, 10.1364/AO.99.099999 . hal01269584

\section{HAL Id: hal-01269584 \\ https://hal.science/hal-01269584}

Submitted on 5 Feb 2016

HAL is a multi-disciplinary open access archive for the deposit and dissemination of scientific research documents, whether they are published or not. The documents may come from teaching and research institutions in France or abroad, or from public or private research centers.
L'archive ouverte pluridisciplinaire HAL, est destinée au dépôt et à la diffusion de documents scientifiques de niveau recherche, publiés ou non, émanant des établissements d'enseignement et de recherche français ou étrangers, des laboratoires publics ou privés. 


\title{
Multilayer four-flux matrix model accounting for directional-diffuse light transfers
}

\author{
L. Simonot, ${ }^{1,}{ }^{*}$ R.D. Hersch, ${ }^{2}$ M. Hébert, ${ }^{3}$ S. MAZAuric ${ }^{3,4}$ \\ ${ }^{1}$ Université de Poitiers, CNRS UPR 3346 Institut Pprime, 11 Boulevard Marie et Pierre Curie, BP 30179,F-86962 Futuroscope Chasseneuil Cedex, \\ France \\ ${ }^{2}$ École Polytechnique Fédérale de Lausanne, Faculté Informatique et Communication, Laboratoire des Systèmes Périphériques, Station 14 , CH- \\ 1015, Lausanne, Switzerland \\ ${ }^{3}$ Université de Lyon, université Jean Monnet de Saint Etienne, CNRS UMR 5516, Laboratoire Hubert Curien, F-42000 Saint Etienne, France \\ ${ }^{4}$ CPE Lyon, Domaine Scientifique de la Doua, 43 boulevard du 11 Novembre 1918 BP 82077, 69616 Villeurbanne Cedex \\ France \\ *Corresponding author: lionel.simonot@univ-poitiers.fr
}

Received XX Month XXXX; revised XX Month, XXXX; accepted XX Month XXXX; posted XX Month XXXX (Doc. ID XXXXX); published XX Month XXXX

The four-flux model is a method to solve light radiative transfer problems in planar, possibly multilayer structures. The light fluxes are modeled as two collimated and two diffuse beams propagating forwards and backwards perpendicularly to the layer stack. In the present contribution, we develop a four-flux model relying on a matrix formalism to determine the reflectance and transmittance factors of stacks of components by knowing those of each individual component. This model is also extended to generate the bidirectional scattering distribution function (BSDF) of the stack by considering an incoming collimated flux in any direction, and by taking into account the directionality of the diffuse fluxes exiting from the material at the border components of the stack. The model is applied to opaque Lambertian backgrounds with flat or rough interface, for which analytical expressions of the BSDF are obtained. (C) 2015 Optical Society of America

OCIS codes: (290.4210) Multiple scattering; (290.1483) BSDF, BRDF and BTDF; (230.4170) Multilayer; (000.3860) Mathematical methods in physics.

http://dx.doi.org/10.1364/AO.99.099999

\section{Introduction}

Predicting the visual appearance of objects, by carrying out an acceptable computation effort, is often a challenge because of the variety of materials and surface finishes, and the complexity of the multiple optical phenomena occurring within the material layers. Accurate prediction is however crucial for the digital design of objects. It requires modeling the multiple reflections and scattering of light at the interfaces and within the turbid media composing the material. A radiometric approach consists in writing the balance for the absorbed and scattered fluxes by introducing the properties of single scattering. In the general tridimensional case, the multiple scattering is mostly solved by Monte Carlo methods where each individual photon event is described with probabilities. For specific material structures, especially stacks of planar layers, one can solve the radiative transfer equation (RTE) [1]. This integro-differential equation takes into account both the spatial position and the orientations of the incident and scattered fluxes. However, as it is complex to solve, many resolution techniques and approximations have been suggested. One common approximation is not to take into account explicitly lateral scattering within the material. Therefore, the equation depends only on one spatial dimension, namely the depth $z$ within the stack. Regarding the angular distribution of light, it can be discretized into $N$ annular solid angles. This $N$-flux model was first proposed by Mudgett and Richards [2] in the case of azimuthally isotropic scattering and generalized by Stamnes et al. [3] under the so-called discrete ordinate method. $N$ can exceed 20 [2] but small $N$ values present the advantage of simple expressions for the reflectance and the transmittance factors. For $N=2$, only two hemispherical fluxes with constant radiance propagate towards positive and negative $z$. In that case, the RTE has analytical solutions, well known as the Kubelka-Munk formulas [4,5]. This twoflux model was also extended to determine the diffuse reflectances and transmittances of stacks of scattering layers [6]. Whereas the KubelkaMunk model is the result of the continuous integration of the RTE, the Kubelka 1954 model can be interpreted as the corresponding discrete summation. The correspondences between the continuous and discrete two-flux approaches have already been discussed $[7,8$,$] .$

However, considering two diffuse fluxes is not possible when the incident light is collimated and a part of it becomes diffuse. The fourflux model with two additional collimated fluxes propagating perpendicularly to the planar layers towards positive and negative $z$ directions improves the reflectance and transmittance predictions in case of collimated illumination. Resolutions of the RTE according to the four-flux approach with various boundary conditions were proposed 
by Beasley et al. [9], Mudgett and Richards [2] and Ishimaru [10]. The formulation proposed by Maheu et al. [11,12] became the main reference when compared with exact calculations for specific cases [13], or after comparisons with Monte Carlo simulations [14]. In their formulation, Maheu et al. introduced an average path length parameter which can take values from 1 for a collimated flux to 2 for a perfect isotropic radiation. They also introduced a forward scattering ratio. The determination of these parameters was discussed by several authors [15-20]. The four-flux model can be expressed by using a matrix formalism as suggested by Rozé et al. in the case of multilayer [21]. Recent formulations [22,23] also enable predicting interface effects. The four-flux model can be used for various scattering systems as illustrated by recent publications [24-27]. However, even if the fourflux approach is much easier to use than more elaborated models, the simplicity of the two-flux approximation is still often preferred. For this reason, intermediate models between two-flux and four-flux have also been proposed [28-30].

In the present study, we use the four-flux model without specifically focusing on the resolution of the RTE. In Section 2, we adopt a matrix formalism to calculate the reflectance and transmittance factors of a superposition of optical components (interfaces and propagating media). This approach can be seen as an extension of the Kubelka model [6] to four fluxes. The main contribution of this study, presented in Section 3, consists in adapting the four-flux matrix model in order to generate families of bidirectional scattering distribution factors (BSDF) by considering an incident collimated flux in any direction and directional diffuse fluxes exiting the material. The bidirectional transfer matrices are described for the particular cases of highly scattering (Lambertian) and of non-scattering (transparent) components in Section 4. We consider flat or rough dielectric interfaces in Section 5. The complete method is finally presented in Section 6 to determine the BSDF in the special cases where a flat or a rough interface is at the top of an opaque Lambertian background.

\section{Four-flux matrix model}

The four-flux model considers a parallel planar structure of material and therefore reduces the radiative transfer equation to a problem with one spatial dimension. It can be presented as a special case of the $\mathrm{N}$-flux model [2] where the radiation field for each position in the stack of layers is composed of two collimated beams $I_{c}$ and $J_{c}$ and two isotropic diffuse beams $I_{d}$ and $J_{d}$. The fluxes propagate perpendicularly to the plane, forwards $\left(I_{c}\right.$ and $\left.I_{d}\right)$ and backwards $\left(J_{c}\right.$ and $\left.J_{d}\right)$.

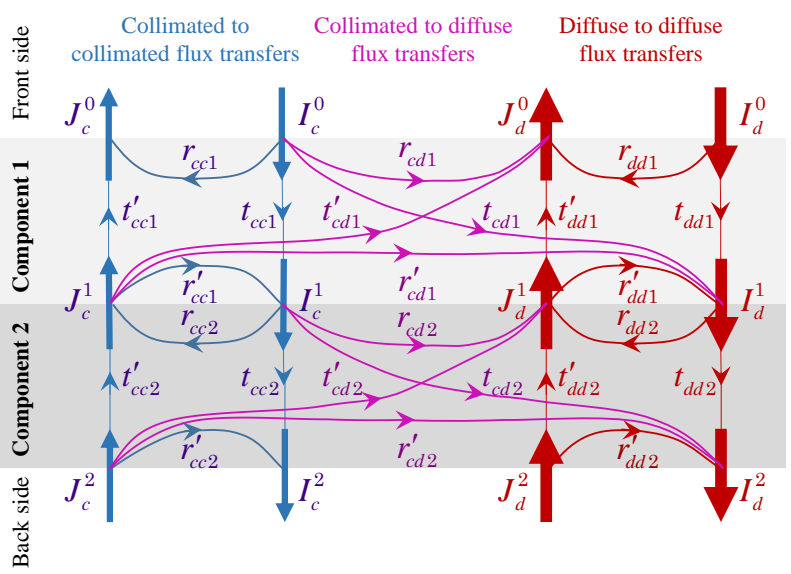

Fig. 1. Flux transfers between two components represented by thin arrows. Bold arrows correspond to fluxes.

A multilayer material can be described as a succession of interfaces and media. Each component of the stack, interface or medium, gives rise to flux transfers: front side reflectance $r$, back side reflectance $r$, forward transmittance $t$ and backward transmittance $t^{\prime}$. They can be collimated-to-collimated (label $c c$ ), diffuse-to-diffuse (label $d d$ ) or collimated-to-diffuse (label $c d$ ) transfers. Figure 1 represents the flux transfers for a stack of two components.

Let us consider the component labelled $k$. The fluxes labelled by superscripts $k$ and $k-1$ are related according to the following equations where, for sake of writing simplicity, we omit the label $k$ in the transfer factors:

$$
\left\{\begin{array}{l}
J_{c}^{k-1}=r_{c c} I_{c}^{k-1}+t_{c c}^{\prime} J_{c}^{k} \\
I_{c}^{k}=t_{c c} I_{c}^{k-1}+r_{c c}^{\prime} J_{c}^{k} \\
J_{d}^{k-1}=r_{c d} I_{c}^{k-1}+t_{c d}^{\prime} J_{c}^{k}+r_{d d} I_{d}^{k-1}+t_{d d}^{\prime} J_{d}^{k} \\
I_{d}^{k}=t_{c d} I_{c}^{k-1}+r_{c d}^{\prime} J_{c}^{k}+t_{d d} I_{d}^{k-1}+r_{d d}^{\prime} J_{d}^{k}
\end{array}\right.
$$

It is easy to verify that these equations express the relationships shown in Figure 1.

\subsection{Matrix formulation}

The system of equations (1) can be presented under two possible matrix equations. The first matrix equation, used to solve the RTE [31] in a multi-angle approach, is shown in Appendix A. We consider here the second matrix equation, which focuses on the transfer nature (collimated-to-collimated, diffuse-to-diffuse and collimated-to-diffuse) and is therefore specific to the four-flux approach:

$$
\left(\begin{array}{cccc}
-r_{c c} & 1 & 0 & 0 \\
t_{c c} & 0 & 0 & 0 \\
-r_{c d} & 0 & -r_{d d} & 1 \\
t_{c d} & 0 & t_{d d} & 0
\end{array}\right)\left(\begin{array}{l}
I_{c}^{k-1} \\
J_{c}^{k-1} \\
I_{d}^{k-1} \\
J_{d}^{k-1}
\end{array}\right)=\left(\begin{array}{cccc}
0 & t_{c c}^{\prime} & 0 & 0 \\
1 & -r_{c c}^{\prime} & 0 & 0 \\
0 & t_{c d}^{\prime} & 0 & t_{d d}^{\prime} \\
0 & -r_{c d}^{\prime} & 1 & -r_{d d}^{\prime}
\end{array}\right)\left(\begin{array}{c}
I_{c}^{k} \\
J_{c}^{k} \\
I_{d}^{k} \\
J_{d}^{k}
\end{array}\right)
$$

The matrix on the left-hand side can be inverted if $t_{c c} t_{d d} \neq 0$. By leftmultiplying both member of Eq. (2) with the inverse of the left-most matrix, we obtain the following equation exhibiting the transfer matrix of the considered component, which is written for convenience under a $2 \times 2$ block form:

$$
\left(\begin{array}{l}
I_{c}^{k-1} \\
J_{c}^{k-1} \\
I_{d}^{k-1} \\
J_{d}^{k-1}
\end{array}\right)=\left(\begin{array}{cc}
\mathbf{M}_{c c} & \mathbf{0}_{2,2} \\
\mathbf{M}_{c d} & \mathbf{M}_{d d}
\end{array}\right)\left(\begin{array}{c}
I_{c}^{k} \\
J_{c}^{k} \\
I_{d}^{k} \\
J_{d}^{k}
\end{array}\right)
$$

where each block is a $2 \times 2$ matrix. The two blocks on the diagonal, corresponding to collimated-to-collimated transfers $(x x=c c)$ and diffuse-to-diffuse transfers $(x x=d d)$, are

$$
\mathbf{M}_{x x}=\frac{1}{t_{x x}}\left(\begin{array}{cc}
1 & -r_{x x}^{\prime} \\
r_{x x} & t_{x x} t_{x x}^{\prime}-r_{x x} r_{x x}^{\prime}
\end{array}\right)
$$

and the left-bottom block, corresponding to collimated-to-diffuse transfers $(c d)$, is

$$
\mathbf{M}_{c d}=\frac{1}{t_{c c} t_{d d}}\left(\begin{array}{cc}
-t_{c d} & r_{c c}^{\prime} t_{c d}-r_{c d}^{\prime} t_{c c} \\
r_{c d} t_{d d}-r_{d d} t_{c d} & t_{c c}\left(t_{c d}^{\prime} t_{d d}-r_{c d}^{\prime} r_{d d}\right)-r_{c c}^{\prime}\left(r_{c d} t_{d d}-r_{d d} t_{c d}\right)
\end{array}\right)
$$

From a given transfer matrix, the reflectances and transmittances of the component (or stack of components) can be obtained provided $\mathbf{M}_{c c}(1,1) \neq 0$ and $\mathbf{M}_{d d}(1,1) \neq 0$. Let us express for example to frontside reflectance and the front-to-back transmittance for the different tupes of transfers. For collimated-to-collimated $(x x=c c)$ and diffuse-todiffuse $(x x=d d)$ transfers, they are given by: 


$$
t_{x x}=\frac{1}{\mathbf{M}_{x x}(1,1)} \text { and } r_{x x}=\frac{\mathbf{M}_{x x}(2,1)}{\mathbf{M}_{x x}(1,1)}
$$

and, for collimated-to-diffuse transfers, they are given by

$$
\begin{gathered}
t_{c d}=\frac{-\mathbf{M}_{c d}(1,1)}{\mathbf{M}_{c c}(1,1) \mathbf{M}_{d d}(1,1)} \\
r_{c d}=\frac{\mathbf{M}_{c d}(2,1)}{\mathbf{M}_{c c}(1,1)}-\frac{\mathbf{M}_{c d}(1,1) \mathbf{M}_{d d}(2,1)}{\mathbf{M}_{c c}(1,1) \mathbf{M}_{d d}(1,1)}
\end{gathered}
$$

\subsection{Matrix multiplication}

In order to obtain the transfer matrix representing a stack of components, the components' individual transfer matrices are multiplied by respecting the stacking order of the components. With two components characterized by matrices $\mathbf{M}_{1}$ and $\mathbf{M}_{2}$, from front to back, the transfer matrix of the two components together is:

$$
\mathbf{M}_{1} \mathbf{M}_{2}=\left(\begin{array}{cc}
\mathbf{M}_{c c 1} \mathbf{M}_{c c 2} & \mathbf{0}_{2,2} \\
\mathbf{M}_{c d 1} \mathbf{M}_{c c 2}+\mathbf{M}_{d d 1} \mathbf{M}_{c d 2} & \mathbf{M}_{d d 1} \mathbf{M}_{d d 2}
\end{array}\right)
$$

Applying the 2x2 matrix multiplication $\mathbf{M}_{x x 1} \mathbf{M}_{x x 2}$ and the formulas (6) yields, for collimated-to-collimated $(x x=c c)$ or diffuse-to-diffuse $(x x=d d)$ transfers, the global transmittances and reflectances of the two component stack:

$$
t_{x x}=\frac{t_{x x 1} t_{x x 2}}{1-r_{x x 1}^{\prime} r_{x x 2}} \text { and } r_{x x}=r_{x x 1}+\frac{r_{x x 2} t_{x x 1} t_{x x 1}^{\prime}}{1-r_{x x 1}^{\prime} r_{x x 2}}
$$

Applying the 2x2 matrix operation $\mathbf{M}_{c d 1} \mathbf{M}_{c c 2}+\mathbf{M}_{d d 1} \mathbf{M}_{c d 2}$ and the formulas (7) yields, for collimated-to-diffuse transfers,

$$
\begin{aligned}
& t_{c d}=\frac{t_{c d 1} t_{d d 2}}{1-r_{d d 1}^{\prime} r_{d d 2}}+\frac{t_{c c 1} t_{c d 2}}{1-r_{c c 1}^{\prime} r_{c c 2}}+\frac{t_{c c 1} t_{d d 2}\left(r_{c d 1}^{\prime} r_{c c 2}+r_{d d 1}^{\prime} r_{c d 2}\right)}{\left(1-r_{c c 1}^{\prime} r_{c c 2}\right)\left(1-r_{d d 1}^{\prime} r_{d d 2}\right)} \\
& r_{c d}=r_{c d 1}+\frac{t_{c d 1} r_{d d 2} t_{d d 1}^{\prime}}{1-r_{d d 1}^{\prime} r_{d d 2}}+\frac{t_{c d 1}^{\prime} r_{c c 2} t_{c c 1}}{1-r_{c c 1}^{\prime} r_{c c 2}}+\frac{t_{c c 1} t_{d d 1}^{\prime}\left(r_{c d 2}+r_{d d 2} r_{c d 1}^{\prime} r_{c c 2}\right)}{\left(1-r_{c c 1}^{\prime} r_{c c 2}\right)\left(1-r_{d d 1}^{\prime} r_{d d 2}\right)}
\end{aligned}
$$

If either collimated-only or diffuse-only fluxes are considered, the model becomes the two-flux model described by its corresponding 2x2 matrices (either $\mathbf{M}_{c c}$ or $\mathbf{M}_{d d}$ ). Similar relations as equations (9) were derived by Stokes [32] in order to predict the specular reflectances and regular transmittances of stacks of glass plates, and later by Kubelka [6] to predict the diffuse reflectances and transmittances of stacks of strongly scattering layers.

Given the complex expressions of the collimated-to-diffuse reflectance and transmittance (Eqs. (10) and (11)), even with two components only, the matrix formalism is much more convenient. However, the matrix computation is valid only when the following condition is satisfied for each component:

$$
t_{c c i} t_{d d i} \neq 0
$$

For example with an opaque component, this condition cannot be satisfied. In these cases, the matrix calculations are first performed with the literal expressions of these transmittances. They are then set to zero at the very final step of the calculation.

\section{Extension of the four-flux matrix model to express the BSDF of component stacks}

In its original expression [11], the four-flux model assumes collimated and isotropic hemispherical diffuse fluxes propagating perpendicular to the stack of layers. In order to define BSDF models from the four-flux matrix method presented in Section 2, some adaptations are needed. The BSDF expresses the bidirectional reflectance and transmittance distribution functions (BRDF and BTDF), for which the incident illumination is assumed to be a unique collimated flux in any direction $\mathbf{i}$ of the upper hemisphere, not only at the normal incidence. Moreover, the diffuse fluxes exiting the material from the border components of the stack are not assumed necessarily Lambertian and can therefore depend on the output direction o of the upper hemisphere for BRDF and of the lower hemisphere for BTDF.

The radiometric definitions and relations used in this section are detailed in the literature, for example in Refs $[33,34]$.

\subsection{BSDF configuration}

According to the definition of the BSDF, the incident illumination is assumed to be a collimated flux in the incident direction $\mathbf{i}$. There is no incident diffuse flux or upward incident flux. In the case of the system represented in Figure 1, this means that $I_{d}^{0}=J_{c}^{2}=J_{d}^{2}=0$. The scattered light is captured in every direction o. Figure 2 explicits the notations.

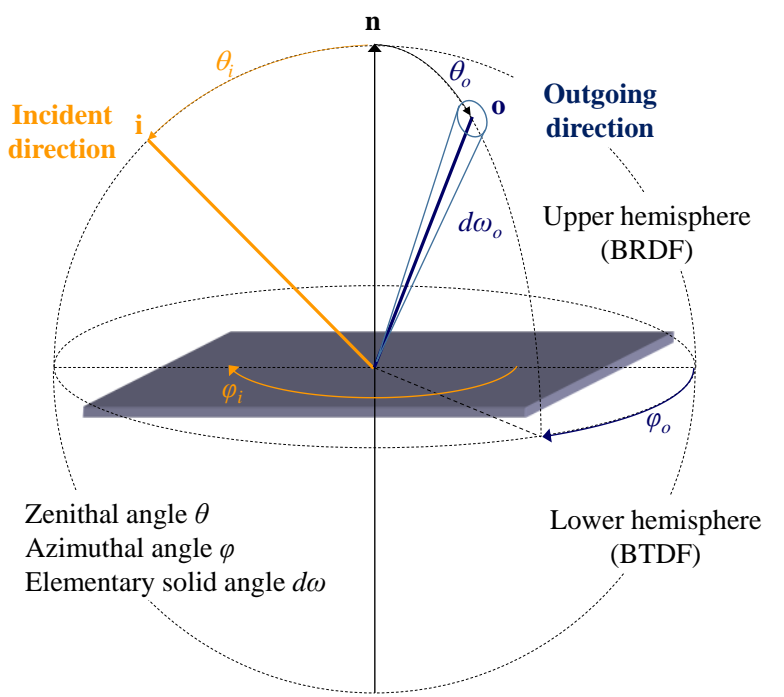

Fig. 2: Useful notations for defining the BSDF.

\subsection{Collimated fluxes related to the incident direction}

The collimated incident beam is defined for a freely chosen orientation i within the upper hemisphere. The collimated fluxes after multiple reflections and transmissions remain within the incident plane. Knowing the different refractive indices, the directions of the downward fluxes are defined from the incident direction $\mathbf{i}$ and by Snell's refraction law. The direction of each upward flux is deduced from the direction of the corresponding downward flux according to Snell's reflection law.

\subsection{Directional diffuse output transfers}

In the original four-flux model, the diffuse fluxes are assumed to be hemispherical with angle-independent radiance (i.e. Lambertian). In our approach, we make an exception for the diffuse fluxes exiting the material from the first or from the last component of the stack. Therefore, the reflectance and transmittance factors to be chosen depend on the position of the component in the stack. Directional light that exits the stack of components is diffused according to the BRDF and the BTDF of the bordering components. Table 1 defines the collimated-to-diffuse and diffuse-to-diffuse transfer factors to be used according to the definitions by Nicodemus et al. [33]. For the sake of simplicity, we specify vector $\mathbf{i}$ only when the incident light is collimated, and/or vector o only when light is captured in one direction (see Tables 1 and 2). 
Table 1: Collimated-to-diffuse and diffuse-to-diffuse reflectance and transmittance factors of a component according to its position in the component stack. The greyed cells are non-useful situations according to the assumptions presented in Section 3.1.

\begin{tabular}{|c|c|c|c|}
\hline & $\begin{array}{c}\text { First } \\
\text { component }\end{array}$ & $\begin{array}{l}\text { Intermediary } \\
\text { component }\end{array}$ & $\begin{array}{c}\text { Last } \\
\text { component }\end{array}$ \\
\hline$r_{c d}$ & $\begin{array}{l}\text { Bidirectional reflectance } \\
\text { factor } r_{c d}(\mathbf{i}, \mathbf{o})\end{array}$ & \multicolumn{2}{|c|}{ Directional-hemispherical reflectance factor $r_{c d}(\mathbf{i})$} \\
\hline$r_{c d}^{\prime}$ & \multicolumn{2}{|c|}{ Directional-hemispherical reflectance factor $r_{c d}^{\prime}(\mathbf{i})$} & \\
\hline$r_{d d}$ & & \multicolumn{2}{|c|}{ Bihemispherical reflectance factor $r_{d d}$} \\
\hline$r_{d d}^{\prime}$ & \multicolumn{2}{|c|}{ Bihemispherical reflectance factor $r_{d d}^{\prime}$} & \\
\hline$t_{c d}$ & \multicolumn{2}{|c|}{ Directional-hemispherical transmittance factor $t_{c d}(\mathbf{i})$} & $\begin{array}{l}\text { Bidirectional transmittance } \\
\quad \text { factor } t_{c d}(\mathbf{i}, \mathbf{o})\end{array}$ \\
\hline$t_{c d}^{\prime}$ & $\begin{array}{l}\text { Bidirectional transmittance } \\
\text { factor } t_{c d}^{\prime}(\mathbf{i}, \mathbf{o})\end{array}$ & $\begin{array}{l}\text { Directional-hemispherical } \\
\text { transmittance factor } t_{c d}^{\prime}(\mathbf{i})\end{array}$ & \\
\hline$t_{d d}$ & & $\begin{array}{l}\text { Bihemispherical transmittance } \\
\text { factor } t_{d d}\end{array}$ & $\begin{array}{l}\text { Hemispherical-directional } \\
\text { transmittance factor } t_{d d}(\mathbf{o})\end{array}$ \\
\hline$t_{d d}^{\prime}$ & $\begin{array}{l}\text { Hemispherical-directional } \\
\text { transmittance factor } t_{d d}^{\prime}(\mathbf{o})\end{array}$ & $\begin{array}{l}\text { Bihemispherical transmittance } \\
\text { factor } t_{d d}^{\prime}\end{array}$ & \\
\hline
\end{tabular}

By knowing the BRDF and the BTDF of a component, we can define its reflectance or transmittance factors according to the formulas given by Table 2 [33]. For a stack of components, according to Table 1, and after the matrix multiplications, one can deduce the BRDF $f_{r}(\mathbf{i}, \mathbf{o})$ and BTDF $f_{t}(\mathbf{i}, \mathbf{o})$ of the stack:

$$
f_{r}(\mathbf{i}, \mathbf{o})=\frac{r_{c d}(\mathbf{i}, \mathbf{o})}{\pi} \text { and } f_{t}(\mathbf{i}, \mathbf{o})=\frac{t_{c d}(\mathbf{i}, \mathbf{o})}{\pi}
$$

where the bidirectional reflectance and transmittance factors, $r_{c d}(\mathbf{i}, \mathbf{o})$ and $t_{c d}(\mathbf{i}, \mathbf{o})$, are given by Eqs (7).

Moreover, in the case where part of the incident collimated flux remains collimated after exiting the border components, the specular reflectance $r_{c c}(\mathbf{i})$ and the regular transmittance $t_{c c}(\mathbf{i})$, given by Eq. (6) with $x x=c c$, have to be added to the BSDF, formally by using Dirac delta functions [35].

Table 2: Expressions of the different reflectance or transmittance factors in function of the BRDF or BTDF $f$, where

$$
\int_{2 \pi} f \cos \theta d \omega=\int_{\theta=0}^{\pi / 2} \int_{\varphi=0}^{\pi / 2} f \cos \theta \sin \theta d \theta d \varphi
$$

\begin{tabular}{|c|c|c|}
\hline Reflectance/transmittance factors & $\begin{array}{c}\text { Directionality } \\
\text { dependency }\end{array}$ & $\begin{array}{c}\text { Expressions as a function } \\
\text { of the BRDF/BTDF } f(\mathbf{i}, \mathbf{o})\end{array}$ \\
\hline $\begin{array}{c}\text { Bidirectional factor } \\
r_{c d}, t_{c d}, t_{c d}^{\prime}\end{array}$ & $(\mathbf{i}, \mathbf{o})$ & $\pi f(\mathbf{i}, \mathbf{o})$ \\
\hline $\begin{array}{c}\text { Directional-hemispherical factor } \\
r_{c d}, r_{c d}^{\prime}, t_{c d}, t_{c d}^{\prime}\end{array}$ & $(\mathbf{i})$ & $\int_{2 \pi} f(\mathbf{i}, \mathbf{o}) \cos \theta_{o} d \omega_{o}$ \\
\hline $\begin{array}{c}\text { Hemispherical-directional factor } \\
t_{d d}, t_{d d}^{\prime}\end{array}$ & $(\mathbf{o})$ & $\int_{2 \pi} f(\mathbf{i}, \mathbf{o}) \cos \theta_{i} d \omega_{i}$ \\
\hline $\begin{array}{c}\text { Bihemispherical factor } \\
r_{d d}, r_{d d}^{\prime}, t_{d d}, t_{d d}^{\prime}\end{array}$ & $\frac{1}{\pi} \int_{2 \pi} \int_{2 \pi} f(\mathbf{i}, \mathbf{o}) \cos \theta_{i} d \omega_{i} \cos \theta_{o} d \omega_{o}$ \\
\hline
\end{tabular}

In contrast with more elaborated models based on the multiangle scattering approach [36], we assume that the diffuse fluxes lose their directionality within the stack. In many systems, this limitation has a weak influence when at least one component of the stack is sufficiently scattering to make the assumption acceptable. But even when it is not the case, the directionality of the collimated fluxes is preserved within the stack, and the directionality of the diffuse fluxes is conserved at the extreme components of the stack. A more restrictive limitation, but intrinsic to the four-flux approach, is the fact that the angular spreading of the collimated fluxes is not rendered.

\section{Transfer matrices for Lambertian and non- scattering components}

Among the optical components, the Lambertian scattering as well as the non-scattering components are interesting limit cases. Their presence in a stack enables important simplifications of the prediction method, especially when the border components of the stack are nonscattering.

(a)

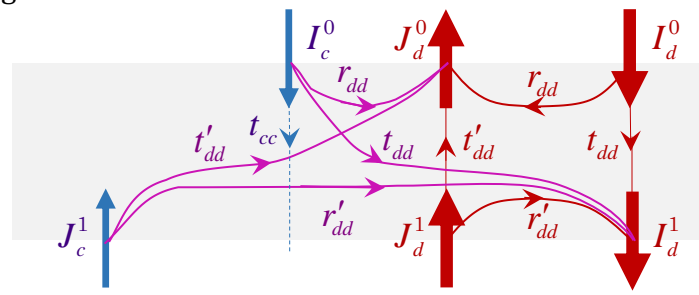

(b)

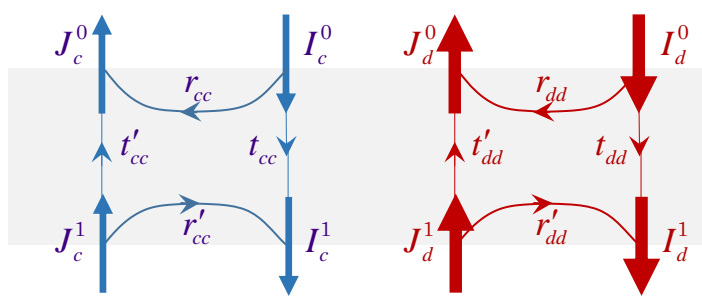

Fig. 3: Flux transfers (a) for a Lambertian component, (b) for a nonscattering component.

\subsection{Lambertian component}

For a Lambertian component, light is uniformly scattered over the hemisphere, independently of the orientation of the incident light. It is worth noting that it is an ideal case [37]. Consequently, an incident collimated light is entirely transformed into diffuse light and $t_{c c}=r_{c c}=0$ (Figure 3a). However, to fulfill the condition (12), we artificially define a transmittance $t_{c c}$, which we will set to zero hereafter, and the following matrix $\mathbf{M}_{c c}$ :

$$
\mathbf{M}_{c c}=\frac{1}{t_{c c}}\left(\begin{array}{ll}
1 & 0 \\
0 & 0
\end{array}\right)
$$

The assumption of a Lambertian component implies that the reflectance and transmittance factors are identical for a collimated or a diffuse incident light flux, and for every output direction $\mathbf{0}$ :

$$
\begin{aligned}
& \left\{\begin{array}{l}
r_{c d}(\mathbf{i}, \mathbf{o})=r_{c d}(\mathbf{i})=r_{d d} \\
t_{c d}(\mathbf{i}, \mathbf{o})=t_{c d}(\mathbf{i})=t_{d d}(\mathbf{o})=t_{d d}
\end{array}\right. \\
& \left\{\begin{array}{l}
r_{c d}^{\prime}(\mathbf{i})=r_{d d}^{\prime} \\
t_{c d}^{\prime}(\mathbf{i}, \mathbf{o})=t_{c d}^{\prime}(\mathbf{i})=t_{d d}^{\prime}(\mathbf{o})=t_{d d}^{\prime}
\end{array}\right.
\end{aligned}
$$

and

Therefore, the transfer matrix for a Lambertian component is independent of its position in the stack according to Table 1. Assuming $t_{c c} t_{d d} \neq 0$, the collimated-to-diffuse transfer matrix (Eq. (5)) can be expressed as

$$
\mathbf{M}_{c d}=\left(\begin{array}{cc}
-\frac{1}{t_{c c}} & -\frac{r_{d d}^{\prime}}{t_{d d}} \\
\frac{r_{d d}}{t_{c c}} & \frac{t_{d d} t_{d d}^{\prime}-r_{d d} r_{d d}^{\prime}}{t_{d d}}
\end{array}\right)
$$

For example, we can define a perfectly Lambertian rough interface or a perfectly Lambertian medium. In the last case, the medium is generally considered as symmetrical and: 


$$
\left\{\begin{array}{l}
r_{c d}=r_{d d}=r_{c d}^{\prime}=r_{d d}^{\prime} \\
t_{c d}=t_{d d}=t_{c d}^{\prime}=t_{d d}^{\prime}
\end{array}\right.
$$

We can check that the superposition of Lambertian components is also Lambertian as its transfer matrix verifies the conditions (14) and (15).

\subsection{Non-scattering component}

Without scattering, the collimated fluxes cannot be transferred into diffuse fluxes (Figure 3b). Therefore:

$$
\mathbf{M}_{c d}=\mathbf{0}_{2,2}
$$

For example, a non-scattering component can be a perfect plane interface or a non-scattering medium. In the last case, the light cannot be reflected by such a medium $\left(r_{c c}=r_{c c}^{\prime}=r_{d d}=r_{d d}^{\prime}=0\right)$ and the matrices Mcc and Mdd are then diagonal.

For the special case of a non-scattering component, the diffuse-todiffuse reflectances or transmittances are the integrals of the collimated beams in all directions of the upper or lower hemisphere, and can then be directly expressed from the corresponding collimatedto-collimated transfer factors:

$$
h_{d d}=\frac{1}{\pi} \int_{\theta_{i}=0}^{\pi / 2} \int_{\varphi_{i}=0}^{2 \pi} h_{c c}(\mathbf{i}) \cos \theta_{i} \sin \theta_{i} d \theta_{i} d \varphi_{i}=\int_{\theta_{i}=0}^{\pi / 2} h_{c c}(\mathbf{i}) \sin 2 \theta_{i} d \theta_{i}
$$

where $h$ is $r, r^{\prime}, t$ or $t^{\prime}$.

It is worth noting that the transfer matrix for the superposition of nonscattering components verifies Equation (18). The resulting component is therefore also non-scattering. The reflectances and transmittances can be determined by operating independently with the $2 \times 2$ transfer matrices $\mathbf{M}_{c c}$ and $\mathbf{M}_{d d}$. However, the diffuse reflectances and transmittances obtained by diffuse-to-diffuse transfer matrix multiplication (Eqs. (9) with $x x=d d$ ) is a crude approximation for non-scattering components. One needs to calculate first the collimated-to-collimated reflectances and transmittances (Eqs. (9) with $x x=c c$ ), and integrating then over the hemisphere (Eq. (19)).

\subsection{Scattering components surrounded by non-scattering components}

When several components are superposed, sub-stacks of nonscattering components are first regrouped and their corresponding transfer matrices are determined. The expressions of their diffuse-todiffuse transmittance factors depend if they are boundary component or not according to Table 1. In the case that the non-scattering substack is the first component, the hemispherical-directional $t_{d d}^{\prime}(\mathbf{o})$ is:

$$
t_{d d}^{\prime}(\mathbf{o})=t_{c c}^{\prime}(\mathbf{o}) / n^{2}
$$

where $n$ is the refractive index ratio between the initial and final media. If it is the last component of the stack, the hemispherical-directional $t_{d d}(\mathbf{o})$ is:

$$
t_{d d}(\mathbf{o})=n^{2} t_{c c}(\mathbf{o})
$$

The factor $1 / n^{2}$ in equation (20), respectively the factor $n^{2}$ in equation (21), is related to the conservation of the optical extent and takes into account the extension of the light beam towards a less refractive medium, respectively the contraction of the light beam towards a more refractive medium [34].

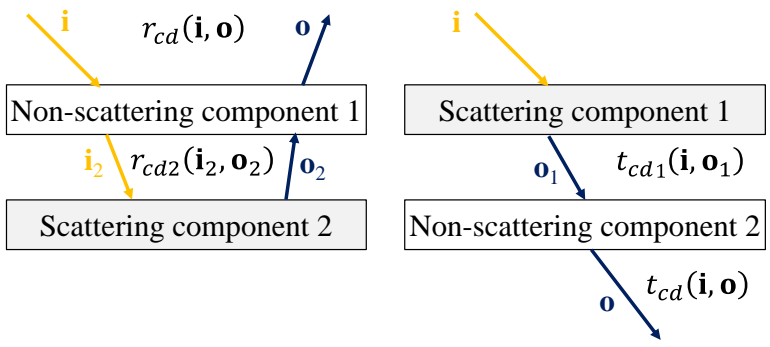

Fig. 4: (a) Bidirectional reflectance factor $r_{c d}(\mathbf{i}, \mathbf{o})$ of a non-scattering component (index 1) on a scattering component (index 2), (b) bidirectional transmittance factor $t_{c d}(\mathbf{i}, \mathbf{o})$ of a scattering component (index 1) on a non-scattering component (index 2)

A non-scattering component presents the advantage to preserve the bidirectional reflectance factor, respectively transmittance factor, when it is the first or the last component. Let us consider a nonscattering component (index 1) on any scattering component (index 2) as represented in Figure 4a. By using equation (11) and the properties of the non-scattering component 1 (Eqs (18) and (20)), the resulting bidirectional reflectance factor $r_{c d}(\mathbf{i}, \mathbf{o})$ can be expressed in terms of the one of the component $2, r_{c d 2}\left(\mathbf{i}_{2}, \mathbf{o}_{2}\right)$ :

$$
r_{c d}(\mathbf{i}, \mathbf{o})=t_{c c 1}(\mathbf{i}) \frac{t_{c c 1}^{\prime}(\mathbf{o})}{n^{2}} \frac{r_{c d 2}\left(\mathbf{i}_{2}, \mathbf{o}_{2}\right)}{\left(1-r_{c c 1}^{\prime}(\mathbf{i}) r_{c c 2}(\mathbf{i})\right)\left(1-r_{d d 1}^{\prime} r_{d d 2}\right)}
$$

where the directions $\mathrm{i}_{2}$ and $\mathrm{o}_{2}$ are related to the directions respectively $i$ and $o$ according to Snell's refraction law.

With similar considerations, we can obtain the bidirectional transmittance factor for any scattering component (index 1) on a nonscattering component (index 2) as represented in Figure 4b:

$$
t_{c d}(\mathbf{i}, \mathbf{o})=\frac{t_{c c 2}(\mathbf{o})}{n^{2}}\left(\frac{t_{c d 1}\left(\mathbf{i}, \mathbf{o}_{1}\right)}{1-r_{d d 1}^{\prime} r_{d d 2}}+\frac{t_{c c 1}(\mathbf{i}) r_{c d 1}^{\prime}(\mathbf{i}) r_{c c 2}(\mathbf{i})}{\left(1-r_{c c 1}^{\prime}(\mathbf{i}) r_{c c 2}(\mathbf{i})\right)\left(1-r_{d d 1}^{\prime} r_{d d 2}\right)}\right)
$$

where the direction $0_{1}$ is related to the outgoing direction 0 according to the Snell's refraction law.

\section{Transfer matrices for flat and rough interfaces}

As the first and the last components mainly influence the BRDF and BTDF of a layered material, the scattering responses of these two components must be analyzed carefully. These border components are most often interfaces. We present the corresponding four-flux matrices for a flat or a rough interface between two dielectric media labeled 0 and 1 , with respective refractive indices $n_{0}$ and $n_{1}$. The relative refractive index of the interface is denoted as $n=n_{1} / n_{0}$.

\subsection{Flat interface}

A flat interface is a non-scattering component, which does not enable any collimated-to-diffuse light transfer. Therefore, the $2 \times 2$ matrix $\mathbf{M}_{c d}$ is a zero matrix. The collimated-to-collimated transfers are given by the Fresnel formulae as functions of the incident direction i. By calling $r_{c c}=R_{01}(\mathbf{i})$ and $t_{c c}=T_{01}(\mathbf{i})$, and by considering 


$$
\left\{\begin{array}{l}
n \sin \left(\theta_{i_{1}}\right)=\sin \left(\theta_{i}\right) \\
t_{c c}=T_{01}(\mathbf{i})=1-R_{01}(\mathbf{i}) \\
r_{c c}^{\prime}=R_{10}\left(\mathbf{i}_{1}\right)=R_{01}(\mathbf{i}) \\
t_{c c}^{\prime}=T_{10}\left(\mathbf{i}_{1}\right)=1-R_{01}(\mathbf{i})
\end{array}\right.
$$

the collimated-to-collimated transfer matrix can be written according to formulas (5) and (24):

$$
\mathbf{M}_{c c}=\frac{1}{T_{01}(\mathbf{i})}\left(\begin{array}{cc}
1 & -R_{01}(\mathbf{i}) \\
R_{01}(\mathbf{i}) & 1-2 R_{01}(\mathbf{i})
\end{array}\right)
$$

Regarding the diffuse-to-diffuse transfer matrix, the bihemispherical reflectance factor $r_{d d}$ is obtained by the angular integration of equation (19) [38]:

$$
r_{d d}=r_{01}=\int_{\theta_{i}=0}^{\pi / 2} R_{01}(\mathbf{i}) \sin 2 \theta_{i} d \theta_{i}
$$

The other bihemispherical reflectance and transmittance factors can then be easily deduced from $r_{d d}$ by the relations:

$$
\left\{\begin{array}{l}
t_{d d}=t_{01}=1-r_{01} \\
t_{d d}^{\prime}=t_{10}=t_{01} / n^{2} \\
r_{d d}^{\prime}=r_{10}=1-t_{10}
\end{array}\right.
$$

These bihemispherical factors only depend on the refractive index ratio $n$ and can be expressed analytically [39].

According to equations (20) and (21), if the flat interface is the first or the last component of the stack, the hemispherical-directional transmittance factors can be calculated as:

$$
\begin{gathered}
t_{d d}^{\prime}(\mathbf{o})=T_{01}(\mathbf{o}) / n^{2} \\
t_{d d}(\mathbf{o})=n^{2} T_{01}(\mathbf{o})
\end{gathered}
$$

\subsection{Rough interface}

Any model describing the BSDF of a rough interface can be used in the four-flux model presented in this article. We adopt the microfacet model described by Walter et al. [35]. The roughness parameter $\sigma$ becomes an additional index for all transfer factors. We assume that the incident collimated flux is completely converted into diffuse fluxes. Hence, the collimated-to-collimated transfers are assumed to be zero (Eq. (14)). The bidirectional reflectance factor is defined as

$$
r_{01 \sigma}(\mathbf{i}, \mathbf{o})=\pi \frac{R_{01}\left(\mathbf{i}, \mathbf{h}_{r}\right) D_{\sigma}\left(\mathbf{h}_{r}\right) G\left(\mathbf{i}, \mathbf{o}, \mathbf{h}_{r}\right)}{4(\mathbf{i} \bullet \mathbf{n})(\mathbf{o} \bullet \mathbf{n})}
$$

and the bidirectional transmittance factor as:

$$
t_{01 \sigma}(\mathbf{i}, \mathbf{o})=\pi \frac{\left|\mathbf{i} \cdot \mathbf{h}_{t}\right|\left|\mathbf{o} \cdot \mathbf{h}_{t}\right|}{(\mathbf{i} \bullet \mathbf{n})(\mathbf{o} \cdot \mathbf{n})} \frac{n_{1}^{2} T_{01}\left(\mathbf{i}, \mathbf{h}_{t}\right) D_{\sigma}\left(\mathbf{h}_{t}\right) G\left(\mathbf{i}, \mathbf{o}, \mathbf{h}_{t}\right)}{\left(n_{0}\left(\mathbf{i} \bullet \mathbf{h}_{t}\right)+n_{1}\left(\mathbf{o} \cdot \mathbf{h}_{t}\right)\right)^{2}}
$$

where $D_{\sigma}$ is the distribution of the microfacet normals, $G$ is a shadowing-masking term, and the directions $\mathbf{h}_{r}$ and $\mathbf{h}_{t}$ are defined as $\mathbf{h}_{r}=(\mathbf{i}+\mathbf{o}) /\|\mathbf{i}+\mathbf{o}\|$ and $\mathbf{h}_{t}=-\left(n_{0} \mathbf{i}+n_{1} \mathbf{o}\right) /\left\|n_{0} \mathbf{i}+n_{1} \mathbf{o}\right\|$.

All entries of the transfer matrix are reflectance and transmittance factors that can be calculated according to the position of the component within the stack (Table 1), and to the angular distribution of the incident flux on both faces (relations of Table 2). For example, the following equation gives the expression of the bihemispherical reflectance factor $r_{01 \sigma}$ as a function of the directional-hemispherical reflectance factor $r_{01 \sigma}(\mathbf{i})$ :

$$
r_{01 \sigma}=\int_{\theta_{i}=0}^{\pi / 2} r_{01 \sigma}(\mathbf{i}) \sin 2 \theta_{i} d \theta_{i}
$$

Appendix B shows the calculated directional-hemispherical and the bihemispherical factors of a rough interface for various roughness parameter values, and presents a way to preserve the energy at the interface.

\section{Flat or rough interface on an opaque Lambertian background}

As case studies, we consider a flat or a rough interface on the top of an opaque Lambertian background (Figures 5a and 5b). These systems involve only two components and the matrix calculations result in compact analytical relations.

(a)

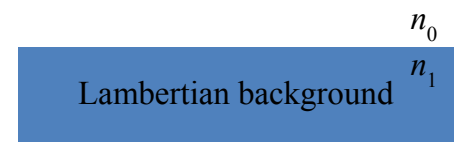

(b)

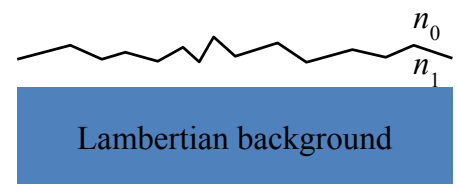

(c)

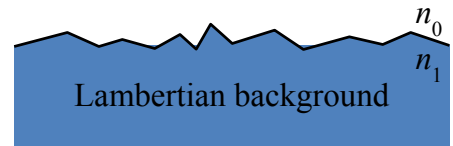

Fig. 5: (a) Perfectly flat interface on a Lambertian background, (b) microfacet rough interface on a Lambertian background, (c) distribution of interfaced Lambertian microfacets.

The calculation consists in multiplying the transfer matrix of the interface described in Section 5 by the transfer matrix of the opaque Lambertian background defined in Section 4.2. As a Lambertian component, the substrate matrix must verify the relations (14) and (16). The background diffuse reflectance is denoted as $\rho=r_{d d}=r_{c d}$. Although the substrate is opaque, we artificially assume that $t_{c c} \neq 0$ and $t_{d d} \neq 0 \quad$ (Eq. (12)) while $t_{c d}=t_{c d}^{\prime}=t_{c c}^{\prime}=t_{d d}^{\prime}=0$ and $r_{c d}^{\prime}=r_{c c}^{\prime}=r_{d d}^{\prime}=0$. The transfer matrices of the opaque Lambertian background can then be written as:

$$
\mathbf{M}_{c c}=\left(\begin{array}{cc}
\frac{1}{t_{c c}} & 0 \\
0 & 0
\end{array}\right), \mathbf{M}_{d d}=\left(\begin{array}{cc}
\frac{1}{t_{d d}} & 0 \\
\frac{\rho}{t_{d d}} & 0
\end{array}\right) \text { and } \mathbf{M}_{c d}=\left(\begin{array}{cc}
0 & 0 \\
\frac{\rho}{t_{c c}} & 0
\end{array}\right)
$$

\subsection{Flat interface on a Lambertian background}

As the first component of the stack, the transfer matrices for the plane interface are (Eqs. (25)-(28)):

$$
\begin{aligned}
\mathbf{M}_{c c} & =\left(\begin{array}{cc}
\frac{1}{T_{01}(\mathbf{i})} & \frac{-R_{01}(\mathbf{i})}{T_{01}(\mathbf{i})} \\
\frac{R_{01}(\mathbf{i})}{T_{01}(\mathbf{i})} & \frac{1-2 R_{01}(\mathbf{i})}{T_{01}(\mathbf{i})}
\end{array}\right), \\
\mathbf{M}_{d d} & =\left(\begin{array}{cc}
\frac{1}{t_{01}} & \frac{-r_{10}}{t_{01}} \\
\frac{r_{01}}{t_{01}} & \frac{t_{01} T_{01}(\mathbf{o}) / n^{2}-r_{01} r_{10}}{t_{01}}
\end{array}\right) \text { and } \mathbf{M}_{c d}=\mathbf{0}_{2,2}
\end{aligned}
$$


The resulting transfer matrix is obtained by multiplying the interface matrix (34) with the background matrix (33). We obtain the following matrices for the stack of the two components:

$$
\begin{gathered}
\mathbf{M}_{c c}=\left(\begin{array}{cc}
\frac{1}{t_{c c} T_{01}(\mathbf{i})} & 0 \\
\frac{R_{01}(\mathbf{i})}{t_{c c} T_{01}(\mathbf{i})} & 0
\end{array}\right), \mathbf{M}_{d d}=\left(\begin{array}{cc}
\frac{1-\rho r_{10}}{t_{01} t_{d d}} & 0 \\
\frac{r_{01}+\rho\left(t_{01} T_{01}(\mathbf{o}) / n^{2}-r_{01} r_{10}\right)}{t_{01} t_{d d}} & 0
\end{array}\right) \\
\text { and } \mathbf{M}_{c d}=\left(\begin{array}{cc}
\frac{-r_{10} \rho}{t_{01} t_{c c}} & 0 \\
\rho \frac{t_{01} T_{01}(\mathbf{o}) / n^{2}-r_{01} r_{10}}{t_{01} t_{c c}} & 0
\end{array}\right)
\end{gathered}
$$

From these matrices, using Eqs. (6) and (7), we can deduce the transfer factors of the interfaced background. Then, by setting $t_{c c}=t_{d d}=0$

(these latter were artificially maintained non-zero), we obtain that the transmittances are zero as expected since the material is opaque. The overall collimated-to-collimated reflectance corresponds to the specular reflectance $R_{01}(\mathbf{i})$ of the flat interface. Finally, the collimatedto-diffuse reflectance $r_{c d}$ of the interfaced background enables deducing the BRDF without its specular component thanks to Eq. (13):

$$
f_{r}(\mathbf{i}, \mathbf{o})=\frac{1}{\pi n^{2}} T_{01}(\mathbf{i}) T_{01}(\mathbf{o}) \frac{\rho}{\left(1-r_{10} \rho\right)}
$$

This analytical relation was firstly obtained by Elias et al. [40] and can be interpreted as a bidirectional extension of the earlier spectral reflectance model by Williams and Clapper [41] for gelatin-based photographic color prints assuming a non-absorbing gelatin layer. The Saunderson correction [42] deals with the same system (flat interface on a Lambertian background) but in the more basic two-flux approach for diffuse light beams. The bidirectional calculation can be easily extended to the case of a stack of non-scattering components instead of a single flat interface either by using the four-flux matrix formalism, or by replacing the interface regular transmittances $T_{01}$ and internal diffuse reflectance $r_{10}$ of Eq. (36) by the equivalent factors of the nonscattering multilayer [43]. The model can be also generalized to nonLambertian background by using Eq. (22), which allows calculating the $\mathrm{BRDF}$ of a flat interface on the top of any substrate whose bidirectional reflectance factor is known.

\subsection{Rough interface on a Lambertian background}

By using the notations introduced in Section 5, the transfer matrices for the rough interface as first component of the stack are written:

$$
\begin{gathered}
\mathbf{M}_{c c}=\left(\begin{array}{cc}
\frac{1}{T_{c c}} & 0 \\
0 & 0
\end{array}\right), \mathbf{M}_{d d}=\left(\begin{array}{cc}
\frac{1}{t_{01 \sigma}} & -\frac{r_{10 \sigma}}{t_{01 \sigma}} \\
\frac{r_{01 \sigma}}{t_{01 \sigma}} & \frac{t_{01 \sigma} t_{10 \sigma}(\mathbf{o})-r_{01 \sigma} r_{10 \sigma}}{t_{01 \sigma}}
\end{array}\right) \text { and } \\
\mathbf{M}_{c d}=\left(\begin{array}{cc}
-\frac{t_{01 \sigma}(\mathbf{i})}{T_{c c} t_{01 \sigma}} & -\frac{r_{10 \sigma}(\mathbf{i})}{t_{01 \sigma}} \\
\frac{t_{01 \sigma} r_{01 \sigma}(\mathbf{i}, \mathbf{o})-t_{01 \sigma}(\mathbf{i}) r_{01 \sigma}}{T_{c c} t_{01 \sigma}} & \frac{t_{10 \sigma}(\mathbf{i}, \mathbf{o}) t_{01 \sigma}-r_{10 \sigma}(\mathbf{i}) r_{01 \sigma}}{T_{c c} t_{01 \sigma}}
\end{array}\right)
\end{gathered}
$$

where $T_{c c}$ is the collimated-to-collimated transmittance of the rough surface, firstly assumed to be non-zero.

After multiplying the rough interface matrix (37) by the opaque Lambertian background matrix (33), we obtain:

$$
\begin{aligned}
& \mathbf{M}_{c c}=\left(\begin{array}{cc}
\frac{1}{T_{c c} t_{c c}} & 0 \\
0 & 0
\end{array}\right), \mathbf{M}_{d d}=\left(\begin{array}{cc}
\frac{1-r_{10 \sigma} \rho}{t_{d d} t_{01 \sigma}} & 0 \\
\frac{r_{01 \sigma}+\rho\left(t_{01 \sigma} t_{10 \sigma}(\mathbf{o})-r_{01 \sigma} r_{10 \sigma}\right)}{t_{d d} t_{01 \sigma}} & 0
\end{array}\right) \\
& \text { and } \mathbf{M}_{c d}=\left(\begin{array}{cc}
-\frac{t_{01 \sigma}(\mathbf{i})}{T_{c c} t_{c c} t_{01 \sigma}}-\frac{r_{10 \sigma} \rho}{t_{c c} t_{01 \sigma}} & 0 \\
\frac{t_{01 \sigma} r_{01 \sigma}(\mathbf{i}, \mathbf{o})-t_{01 \sigma}(\mathbf{i}) r_{01 \sigma}}{T_{c c} t_{c c} t_{01 \sigma}}+\rho \frac{t_{01 \sigma} t_{10 \sigma}(\mathbf{o})-r_{01 \sigma} r_{10 \sigma}}{t_{c c} t_{01 \sigma}} & 0
\end{array}\right)
\end{aligned}
$$

From these matrices, using Eqs. (7) and (13), and by setting $T_{c c}=t_{c c}=t_{d d}=0 \quad$ (artificially maintained non-zero during the calculations), we can deduce the corresponding BRDF with no additional specular term:

$$
f_{r}(\mathbf{i}, \mathbf{o})=\frac{1}{\pi}\left(r_{01 \sigma}(\mathbf{i}, \mathbf{o})+t_{01 \sigma}(\mathbf{i}) t_{10 \sigma}(\mathbf{o}) \frac{\rho}{1-r_{10 \sigma} \rho}\right)
$$

The first term of Eq. (39) is the BRDF of the rough interface (single scattering). The second term is due to the multiple reflections between the inner face of the rough interface and the Lambertian background. As far as we could see in the literature, such analytical expression for this system has never been published. However, as explained in Appendix B, usual microfacet models strongly underestimate the internal diffuse reflectance $r_{10 \sigma}$. To compensate for this energy loss at the interface, we assume this reflectance to be independent of the roughness (see $r_{10 \sigma} \approx 1-t_{10 \sigma}$ in figure B2-b). Consequently, we apply Equation (39) by replacing $r_{10 \sigma}$ by the internal diffuse reflectance $r_{10}$ of a flat interface.

\subsection{Interfaced Lambertian facets}

It is interesting to compare the configuration described by equation (39) and Figure $5 b$ with the one described by Figure $5 c$, developed in a previous work [44], with the same microfacet slope distribution. The corresponding BRDF can be written as:

$$
\begin{aligned}
f_{r}(\mathbf{i}, \mathbf{o})= & \frac{r_{01 \sigma}(\mathbf{i}, \mathbf{o})}{\pi}+\frac{\rho}{\pi\left(1-r_{10} \rho\right)} \frac{1}{n^{2}(\mathbf{i} \bullet \mathbf{n})(\mathbf{o} \bullet \mathbf{n})} \\
& \int_{2 \pi} T_{01}(\mathbf{i}, \mathbf{m}) T_{01}(\mathbf{o}, \mathbf{m}) D_{\sigma}(\mathbf{m}) G(\mathbf{i}, \mathbf{o}, \mathbf{m})(\mathbf{i} \bullet \mathbf{m})(\mathbf{o} \bullet \mathbf{m}) d \omega_{m}
\end{aligned}
$$

where the integral sums up the radiances related to every microfacet normal $\mathbf{m}$ in the hemisphere.

It can be first noted that both models are equivalent for a flat interface $(\sigma=0)$ with the expression given in equation (36). Moreover, the first term $r_{01 \sigma}(\mathbf{i}, \mathbf{o})$, due to surface scattering, is similar for both equations (39) and (40). It corresponds to a Cook-Torrance-like specular lobe [45] whose expression is given in equation (30). We therefore focus the comparison on the second term of equations (39) and (40), due to volume scattering.

When a Lambertian background has a flat interface, its volume BRDF trends rapidly towards zero at grazing incident angles (see Figure 6 for $\sigma=0$ ). This effect is reduced when considering a rough interface superposed over the Lambertian background (Figure 6a). The resulting volume $\mathrm{BRDF}$ tends to the one of a Lambertian reflector when the roughness increases. This BRDF is azimuthally isotropic, which is not the case for the distribution of interfaced Lambertian facets (Figure 6b). The variation of the volume BRDF depends on the observation angle: it decreases when observation is the part of the hemisphere containing the specular direction $\left(\theta_{0}>0\right.$ in Figure $\left.6 \mathrm{~b}\right)$ but it 
increases for grazing angles towards the backscattering direction $\left(\theta_{0}<0\right.$ in Figure 6b). This difference between both models is striking even when there is no refractive index change $(n=1)$ between media. In that specific case, the figure $5 \mathrm{~b}$ (Eq. (39)) is equivalent to the flat Lambert background while the figure $5 \mathrm{c}$ (Eq. (40)) corresponds to a distribution of Lambertian microfacets, and is therefore equivalent to an Oren-Nayar-like model [46].

(a)

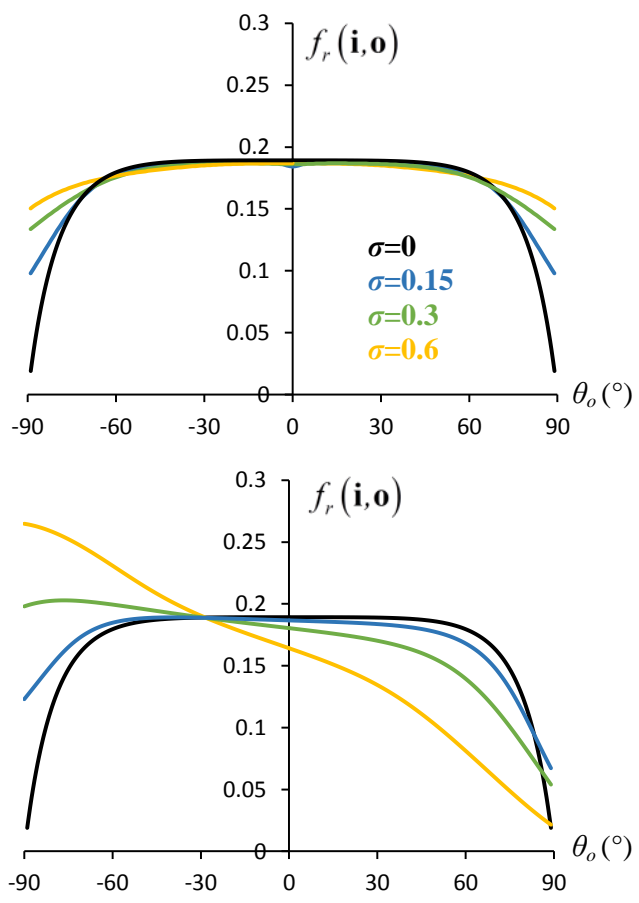

Fig. 6: Volume BRDF in the incident plane, assuming a Beckmann distribution for $D_{\sigma}$ and the corresponding Smith shadowing-masking function for $G[47,48]$, with $\theta_{i}=60^{\circ}$ (backscattering direction at $\theta_{o}=-60^{\circ}$ and specular direction at $\left.\theta_{o}=60^{\circ}\right), n=1.5$ and different roughness parameters $\sigma$, (a) rough interface on a Lambertian background (second term of equation (39) with $r_{10 \sigma}=r_{10}$ ), (b) distribution of interfaced Lambertian facets (second term of equation (40)).

\section{Conclusion}

The methodology presented in this article opens new perspectives to solve radiative transfer problems. We adapt the four-flux model by describing a material as a stack of discrete components. We introduce a $4 \times 4$ transfer matrix that described for each component of the stack, collimated-to-collimated, diffuse-to-diffuse and collimated-to-diffuse flux transfers both in transmittance and reflectance. Stacks of components are built by carrying out the corresponding matrix multiplications. Special matrices describe a Lambertian or a nonscattering component, a flat or a rough interface, and a border component given by its BRDF and BTDF. By construction, the $4 \times 4$ transfer matrix can be reduced to $2 \times 2$ matrices, and the two-flux models for either collimated-only or diffuse-only beams are special cases of this four-flux model. The use of four-flux is justified as soon as at least one component induces collimated to diffuse light transfers, and particularly for translucent materials where the two-flux models fail. We also extend the four-flux to obtain the BSDF of multilayer systems. A restriction of the model is the directionality loss of the diffuse fluxes within the stack. However, in most common cases, this limitation has a negligible impact, because the model accounts for the directionality of the collimated fluxes at every position within the stack, and for the directionality of the diffuse fluxes for the most external scattering components of the stack. Another restriction is that the four- flux approach does not describe the progressive angular broadening of the collimated beams. However, the method is easy to use, offers compact matrix expressions and quick computations. For computer graphics, it allows to generate families of virtual BSDF for a superposition of components knowing the BSDF of each component.

We apply the method for a flat and a rough interface on an opaque Lambertian background. Although systems with only two components are relatively basic, they offer a wide variety of physical based reflectance models from matte to glossy materials. We intend to apply this methodology to more complex systems, where the compact matrix formalism will be an attractive feature.

\section{Appendix A: Another transfer matrix}

The transfer matrix chosen in the article focuses on the nature of the light transfers (labelled $c c, d d$ or $c d$ ). After rearrangement of the flux order for the vectors, another matrix relation, alternative to relation (2) , can be obtained:

$$
\left(\begin{array}{cccc}
t_{c c} & 0 & 0 & 0 \\
t_{c d} & t_{d d} & 0 & 0 \\
-r_{c c} & 0 & 1 & 0 \\
-r_{c d} & -r_{d d} & 0 & 1
\end{array}\right)\left(\begin{array}{l}
I_{c}^{k-1} \\
I_{d}^{k-1} \\
J_{c}^{k-1} \\
J_{d}^{k-1}
\end{array}\right)=\left(\begin{array}{cccc}
1 & 0 & -r_{c c}^{\prime} & 0 \\
0 & 1 & -r_{c d}^{\prime} & -r_{d d}^{\prime} \\
0 & 0 & t_{c c}^{\prime} & 0 \\
0 & 0 & t_{c d}^{\prime} & t_{d d}^{\prime}
\end{array}\right)\left(\begin{array}{c}
I_{c}^{k} \\
I_{d}^{k} \\
J_{c}^{k} \\
J_{d}^{k}
\end{array}\right)
$$

By introducing the $2 \times 2$ matrices $\mathbf{T}, \mathbf{R}, \mathbf{T}^{\prime}$ and $\mathbf{R}^{\prime}$, the matrix relation can be rewritten by block:

$$
\left(\begin{array}{cc}
\mathbf{T} & \mathbf{0}_{2,2} \\
-\mathbf{R} & \mathbf{1}_{2,2}
\end{array}\right)\left(\begin{array}{c}
I_{c}^{k-1} \\
I_{d}^{k-1} \\
J_{c}^{k-1} \\
J_{d}^{k-1}
\end{array}\right)=\left(\begin{array}{cc}
\mathbf{1}_{2,2} & -\mathbf{R}^{\prime} \\
\mathbf{0}_{2,2} & \mathbf{T}^{\prime}
\end{array}\right)\left(\begin{array}{c}
I_{c}^{k} \\
I_{d}^{k} \\
J_{c}^{k} \\
J_{d}^{k}
\end{array}\right)
$$

By assuming $t_{c c} t_{d d} \neq 0$, the matrix of the left side is inverted and leftmultiplied to the matrix of the right side:

$$
\left(\begin{array}{l}
I_{c}^{k-1} \\
I_{d}^{k-1} \\
J_{c}^{k-1} \\
J_{d}^{k-1}
\end{array}\right)=\mathbf{M}\left(\begin{array}{c}
I_{c}^{k} \\
I_{d}^{k} \\
J_{c}^{k} \\
J_{d}^{k}
\end{array}\right)
$$

$$
\text { with } \begin{aligned}
\mathbf{M} & =\left(\begin{array}{cc}
\mathbf{T}^{-1} & \mathbf{0}_{2,2} \\
\mathbf{R T}^{-1} & \mathbf{1}_{2,2}
\end{array}\right)\left(\begin{array}{cc}
\mathbf{1}_{2,2} & -\mathbf{R}^{\prime} \\
\mathbf{0}_{2,2} & \mathbf{T}^{\prime}
\end{array}\right) \\
& =\left(\begin{array}{cc}
\mathbf{T}^{-1} & -\mathbf{T}^{-1} \mathbf{R}^{\prime} \\
\mathbf{R} \mathbf{T}^{-1} & \mathbf{T}^{\prime}-\mathbf{R T}^{-1} \mathbf{R}^{\prime}
\end{array}\right)=\left(\begin{array}{ll}
\mathbf{M}_{11} & \mathbf{M}_{12} \\
\mathbf{M}_{21} & \mathbf{M}_{22}
\end{array}\right)
\end{aligned}
$$

Given the 2x2 block matrices $\mathbf{M}_{i j}$ of the transfer matrix, the following matrix relations can be obtained:

$$
\left\{\begin{array}{l}
\mathbf{R}=\mathbf{M}_{21} \mathbf{M}_{11}^{-1} \\
\mathbf{T}=\mathbf{M}_{11}^{-1} \\
\mathbf{R}^{\prime}=-\mathbf{M}_{11}^{-1} \mathbf{M}_{12} \\
\mathbf{T}^{\prime}=\mathbf{M}_{12}-\mathbf{M}_{21} \mathbf{M}_{11}^{-1} \mathbf{M}_{12}
\end{array}\right.
$$

This transfer-matrix formalism was classically used to solve the radiative transfer equation with $N \mathrm{x} N$ block matrices [31]. Let us note that both matrix expressions (Eqs. (2) and (41)) are related by permutation matrices. 


\section{Appendix B: Energy preservation for a microfacet rough interface}

We consider a rough interface between two media with $n=1.5$, whose bidirectional transfer factors are given by the equations (30) and (31). The function $D_{\sigma}$ is the Beckmann distribution and the corresponding shadowing-masking function $G$ is the one described by Smith [47] and generalized by Bourlier et al. [48]. Figure B1 shows the different directional-hemispherical factors. As expected, the rougher the surface is, the more Lambertian these resulting factors are. The differences with the flat interface $(\sigma=0)$ are more pronounced at grazing incident angles from the less to the more refractive medium (Figure B1a). The differences are much more important in the opposite direction due to the effect of total reflection for a large part of the incident hemisphere (Figure B1b).
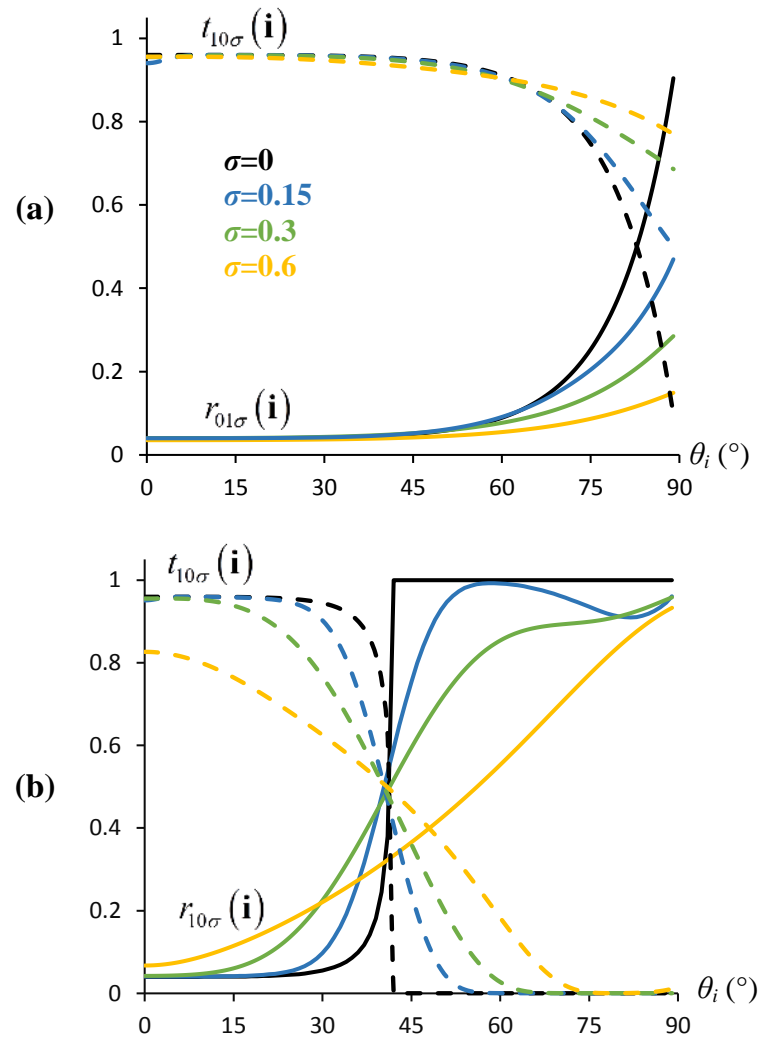

Figure B1: Directional-hemispherical reflectance (solid lines) or transmittance (dashed lines) factors of rough interfaces as a function of the incident angle $\theta_{i}$ for different roughness parameters $\sigma$ with $n=1.5$ (a) from medium 0 to medium 1 , (b) from medium 1 to medium 0.

From relations similar to equation (32), the different bihemispherical factors can be calculated and are represented in figure B2 in terms of the roughness parameter. The conservation of energy should give $r+t=1$. This is however not the case because the microfacet models do not account for interactions with multiple facets. The energy loss increases steadily with the roughness parameter. It is relatively weak from medium 0 to medium 1 ( $3 \%$ loss for $\sigma=0.6)$. It has a larger impact on the diffuse transmittance $t_{01 \sigma}$ than on the diffuse reflectance $r_{01 \sigma}$ (Figure B2a). From medium 1 to medium 0, the loss is much more important ( $20 \%$ loss for $\sigma=0.6$ ). Due to the total reflections, it mainly impacts the diffuse reflectance $r_{10 \sigma}$ (Figure B2b). Let us note that because of energy loss, $r_{01 \sigma}$ continuously decreases with roughness parameter while $t_{10 \sigma}$ is almost constant.

As a physical description of the interactions with multiple facets seems to be very complex, these effects are mostly ignored, or empirically corrected. For example, Jakob et al. [36] suggest to reintroduce the energy loss as a diffuse radiation in reflection and transmission so that the energy is conserved. With the same idea, we suggest to firstly calculate $r_{01 \sigma}$, respectively $t_{10 \sigma}$, and then deduce $t_{01 \sigma}=1-r_{01 \sigma}$, respectively $r_{10 \sigma}=1-t_{10 \sigma}$, to preserve energy conservation. The internal diffuse reflectance $r_{10 \sigma}$ can then be considered as independent of the roughness, and can be approximated by the internal diffuse reflectance $r_{10}$ of a flat interface.

(a)

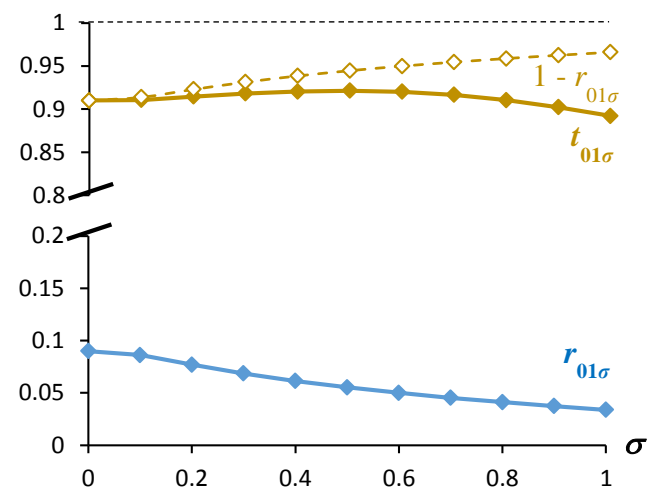

(b)

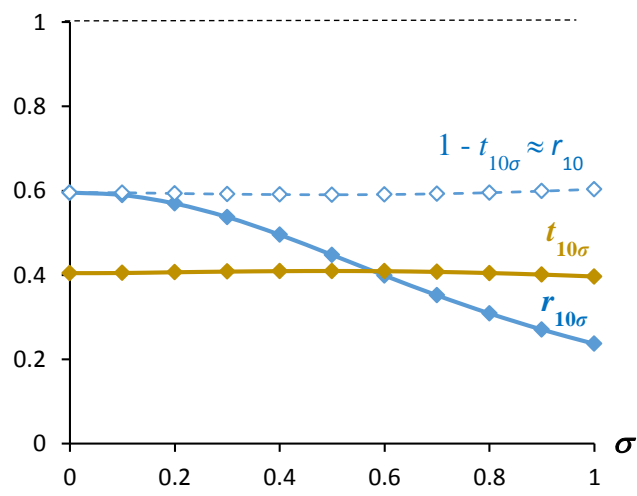

Fig. B2: Bihemispherical factors of a rough interface in terms of the roughness parameter $\sigma$ with $n=1.5$ (a) from medium 0 to medium 1, (b) from medium 1 to medium 0 .

Acknowledgment. L. Simonot thanks the Poitou-Charentes region for founding a visiting followship at the Laboratoire des Systèmes Périphériques in Lausanne from June to August 2015.

\section{References}

1. S. Chandrasekhar, Radiative transfert, Dover, New-York (1960).

2. P.S. Mudgett and L.W. Richards, "Multiple scattering calculations for technology," Appl. Opt. 10, 1485-1502 (1971).

3. K. Stamnes, S. Chee Tsay, W. Wiscombe and K. Jayaweera, "Numerically stable algorithm for discrete-ordinate-method radiative transfer in multiple scattering and emitting layer media," Appl. Opt. 27 2502-2510 (1988).

4. P. Kubelka and F. Munk, "Ein Beitrag zur Optik der Farbanstriche," Zeitschrift für technische Physik 12, 593-601 (1931).

5. P. Kubelka, "New contributions to the optics of intensely light-scattering material, part I," J. Opt. Soc. Am. 38, 448-457 (1948).

6. P. Kubelka, "New contributions to the optics of intensely light-scattering material, part II. Non-homogeneous layers," J. Opt. Soc. Am. 44, 330334 (1954).

7. M. Vöge and K. Simon, "The Kubelka-Munk and Dyck paths," Journal of Statistical Mechanics: Theory and Experiment, P02018 (2007).

8. M. Hébert and J.-M. Becker, "Correspondence between continuous and discrete two-flux models for reflectance and transmittance of diffusing layers," J. Opt. A: Pure Appl. Opt. 10, 035006 (2008). 
9. J.K. Beasley, J.T. Atkins and F.W. Billmeyer, "Scattering and absorption in turbid media," in Electromagnetic scattering Eds R.L. Rowell and R.S. Stein, Gordon and Breach, New York (1967) 765-785.

10. A. Ishimaru, Wave Propagation and Scattering in Random Media, Academic Press, New York (1978).

11. B. Maheu, J.N. Le Toulouzan and G. Gouesbet, "Four-flux models to solve the scattering transfer equation in terms of Lorenz-Mie parameters," Appl. Opt. 23, 3353-3362 (1984).

12. B. Maheu and G. Gouesbet, "Four-flux models to solve the scattering transfer equation. Special cases," Appl. Opt. 25, 1122-1228 (1986).

13. G.A. Niklasson, "Comparison between four flux theory and multiple scattering theory," Appl. Opt. 26, 4033-4036 (1987).

14. B. Maheu, J.P. Briton and G. Gouesbet, "Four-flux model and a Monte Carlo code: comparisons between two simple and complementary tools for multiple scattering calculations," Appl. Opt. 28, 22-24 (1989).

15. Y. P. Wang, S. W. Zheng and K. F. Ren, "Four-flux model with adjusted average crossing parameter to solve the scattering transfer equation," Appl. Opt. 28, 24-26 (1989).

16. W.E. Vargas and G.A. Niklasson, "Forward average path-length parameter in four-flux radiative transfer models," Appl. Opt. 36, 37353738 (1997)

17. W.E. Vargas and G.A. Niklasson, "Generalized method for evaluating scattering parameters used in radiative transfer models," J. Opt. Soc. Am. A 14, 2243-2252 (1997)

18. W.E. Vargas, "Generalized four-flux radiative transfer model average path-length parameter in four-flux radiative transfer models," Appl. Opt. 37, 2615-2623 (1998).

19. C.A. Arancibia-Bulnes and J.C. Ruiz-Suarez, "Average path-length parameter of diffuse light in scattering media," Appl. Opt. 38, 18771883 (1997).

20. C. Rozé, T. Girasole, G. Gréhan, G. Gouesbet and B. Maheu, “Average crossing parameter and forward scattering ratio values in four-flux model for multiple scattering media," Opt. Commun. 194, 251-263 (2001).

21. C. Rozé, T. Girasole and A.G. Tafforin, "Multilayer four-flux model of scattering, emitting and absorbing media," Atmos. Environ. 35, 51255130 (2001).

22. N. Dong, J. Ge and Y. Zhang, "Four-flux Kubelka-Munk model of the light reflectance for printing of rough surface," Proc. of SPIE-IS\&T Electronic Imaging 7241 (2009).

23. M. Hébert, R.D. Hersch and P. Emmel, "Fundamentals of Optics and Radiometry for Color Reproduction," in Handbook of Digital Imaging, Ed. M. Kriss, Wiley, New York, John Wiley \& Sons (2015) 1021-1077.

24. F. El Haber, X. Rocquefelte, C. Andraud, B. Amrani, S. Jobic, O. Chauvet and $\mathrm{G}$. Froyer, "Prediction of the transparency in the visible range of $\mathrm{X}$ ray absorbing nanocomposites built upon the assembly of $\mathrm{LaF}_{3}$ or $\mathrm{LaPO}_{4}$ nanoparticles with poly(methyl methacrylate)," J. Opt. Soc. Am. B 29, 305-311 (2012)

25. S. Bayou, M. Mouzali, F. Aloui, L. Lecamp and P. Lebaudy, "Simulation of conversion profiles inside a thick dental material photopolymerized in the presence of nanofillers," Polymer journal 45, 863-870 (2013).

26. K. Laaksonen, S.-Y. Li, S.R. Puisto, N.K.J. Rostedt, T. Ala-Nissila, C.G. Granqvist, R.M. Nieminen and G.A. Niklasson, "Nanoparticles of $\mathrm{TiO}_{2}$ and $\mathrm{VO}_{2}$ in dielectric media: Conditions for low optical scattering, and comparison between effective medium and four-flux theories," Solar energy materials \& solar cells 130, 132-137 (2014).

27. L. Wang, J.I. Eldridge and S.M. Guo, "Comparison of different models for the determination of the absorption and scattering coefficients of thermal barrier coatings," Acta Materialia 64, 402-410 (2014).

28. J.W. Ryde, "The scattering of light by turbid media - Part 1," Proc. Roy Soc. (London) A131, 451-464 (1931).

29. H. Pauli and D. Eitel, "Comparison of Different Theoretical Models of Multiple Scattering for Pigmented Media," Colour 73, $423-426$ (1973).

30. A.B. Murphy, "Modified Kubelka-Munk model for calculation of the reflectance of coatings with optically-rough surfaces," J. Phys. D: Appl. Phys. 39, 3571-3581 (2006).

31. R. Aronson and D.L. Yarmush, "Transfer-matrix method for gamma-ray and neutron penetration," J. Math. Phys. 7, 221-237 (1966).
32. G.G. Stokes, "On the intensity of the light reflected from or transmitted through a pile of plates," Proc. of the Royal Society of London 11, 545556 (1860).

33. F.E. Nicodemus, J.C. Richmond, J. J. Hsia, I. W. Ginsber and T. Limperis, "Geometrical consideration and nomenclature for reflectance," J. Res. Natl. Bur. Stand. 160, 1-52(1977).

34. M. Hébert and P. Emmel, "Two-flux and multiflux matrix model for color surface", in Handbook of Digital Imaging, Ed. M. Kriss, John Wiley \& Sons (2015) 1233-1277.

35. B. Walter, S.R. Marschner, H. Li and K.E. Torrance, "Microfacet models for refraction through rough surfaces," in Proceeding of Eurographics Symposium on Rendering (2007) 195-206.

36. W. Jakob, E. d'Eon, O. Jakob and S. Marschner, „A comprehensive framework for rendering layered materials," ACM Transactions on Graphics 33, 118, 1-12 (2014).

37. A. Kienle and F. Forschum, " 250 years Lambert surface: does it really exist," Opt. Express 19, 3881-3889 (2011).

38. D.B. Judd, "Fresnel reflection of diffusely incident light," Journal of the National Bureau of Standards 29, 329-332 (1942).

39. R. Molenaar, J.J. ten Bosch and J.R. Zijp, "Determination of KubelkaMunk scattering and absorption coefficient," Appl. Opt. 38, 2028-2860 (1999).

40. M. Elias, L. Simonot and M. Menu, Bidirectional reflectance of a diffuse background covered by a partly absorbing layer, Opt. Commun. 191, 17 (2001)

41. F.C. Williams and F.R. Clapper, "Multiple Internal Reflections in Photographic Color Prints," J. Opt. Soc. Am. 43, 595-597 (1953).

42. J.L. Saunderson, "Calculation of the color pigmented plastics," J. Opt. Soc. Am. 32, 727-736 (1942)

43. L. Simonot, M. Hébert and R. Hersch, "Extension of the WilliamsClapper model to stacked nondiffusing colored coatings with different refractive indices," J. Opt. Soc. Am. A 23, 1432-1441 (2006).

44. L. Simonot, "A photometric model of diffuse surfaces described as a distribution of interfaced Lambertian facets," Appl. Opt. 48, 5793-5801 (2009)

45. R. Cook and K. Torrance, "A reflectance model for computer graphics," Computer Graphics (SIGGRAPH '81 Proceedings) 15, 301-316 (1981).

46. M. Oren and S.K. Nayar, "Generalization of the Lambertian model and implications for machine vision," Int. J. Comput. Vision 14 227-251 (1995)

47. B.G. Smith, "Geometrical shadowing of a random rough surface," IEEE Trans. Ant. Prop. 15, 668-671 (1967)

48. C. Bourlier, G. Berginc and J. Saillard, "One and two-dimensional shadowing functions for any height and slope stationary uncorrelated surface in the monostatic and bistatic configurations," IEEE Trans. Ant. Prop. 50, 312-324 (2002). 